\title{
Pulsed Radiofrequency of the Vagal Nerve for Tinnitus -A Case-Study
}

Henk M. Koning ${ }^{1 *}$

Frits J. van Hemert ${ }^{2}$

\begin{abstract}
Tinnitus Background: Vagal nerve stimulation is a promising therapy for tinnitus. Pulsed radiofrequency is a minimally invasive neuromodulator technique that could be applied repeatedly without damage to neural tissue. In this case-study we document the use of pulsed radiofrequency of the vagal nerve as a successful therapy without harm for patients with tinnitus.

Methods: A 22-gauge, $60 \mathrm{~mm}$-long needle with a $5 \mathrm{~mm}$ active tip was placed percutaneously at the inner side of the tragus. The auricular branch of the vagal nerve was subjected to pulsed radiofrequency at $42 \mathrm{~V}, 2 \mathrm{~Hz}$, and 10 milliseconds for 10 minutes.

Results: Pulsed radiofrequency of the vagal nerve can reduce tinnitus and improve hearing in a patient with severe tinnitus and hearing loss. The intensity of the tinnitus was reduced to $5 \%$ of the original intensity at the left side and to $20 \%$ at the right side. An improved hearing of 20 to $30 \mathrm{~dB}$ from $250 \mathrm{~Hz}$ to $8 \mathrm{kHz}$ in the left ear and from $250 \mathrm{~Hz}$ to $2 \mathrm{kHz}$ in the right ear was objectivated in the audiogram.
\end{abstract}

Conclusions: This case-study document that PRF of the vagal nerve can reduce tinnitus and improve hearing in a patient with severe tinnitus and hearing loss. However, further research of PRF of the vagal nerve in tinnitus patients is needed.

Keywords: Tinnitus, Hearing loss, Vagal nerve stimulation, Pulsed radiofrequency 


\section{INTRODUCTION}

Vagal nerve stimulation (VNS) is a promising therapy for tinnitus ${ }^{1}$. Instead of using an electrode surgically implanted around the left cervical vagal nerve, the auricular branch of vagal nerve (ABVN) can be used to prevent side effects and high costs ${ }^{2,3}$. Stimulating the ABVN has a similar effect on the central auditory pathway of the vagal nerve as the implanted $\mathrm{VNS}^{3,4}$. Transcutaneous stimulation of the ABVN has been introduced, but there is a need for another method of treatment with much longer lasting effect and also with minor side effects ${ }^{3}$. Pulsed radiofrequency (PRF) is a minimally invasive neuromodulator technique which can elicit a long-term effect and can be used again and again without harm to neural tissue ${ }^{5,6}$. In this case-study we document the use of PRF of the vagal nerve as a successful therapy without harm for patients with tinnitus.

\section{CASE REPORT}

A 44-year-old female came to our clinic for therapy of her devastating tinnitus. She presented herself with bilateral tinnitus, with predominance on the left side. Five years ago, a bone anchored hearing aid was placed to overcome her severe hearing loss. She had also serious cervical complaints, postural instability, and dizziness. The mean tinnitus was scored as $100 \mathrm{~mm}$ and the mean cervical pain as 95 on a scale of $0-100 \mathrm{~mm}$. She was exhausted and wanted to live no more.

Already, she was diagnosed with chronic otitis media on both sides for which she underwent bilateral mastoidectomy and bilateral myringoplasty. The intensity of tinnitus was not reduced with medication, such as betahistine, cinnarizine, pregabaline. Further medication included escitalopram, an anti-depressant. Physical assessment demonstrated a decreased rotation of her cervical spine and painful trapezial muscles.

The patient gave informed permission to the PRF of the vagal nerve. The operation was carried out by an experienced anesthesiologist and it occurred without sedation. The patient laid down in prone position on a table. After decontamination of the skin, a 22-gauge, $60 \mathrm{~mm}$-long needle with a $5 \mathrm{~mm}$ active tip was placed percutaneously at the inner side of the left tragus. The needle position was checked and then the ABVN was treated with pulsed radiofrequency at $42 \mathrm{~V}, 2 \mathrm{~Hz}$, and 10 milliseconds for 10 minutes. Postoperative, the patient was monitored for 30 minutes. Her vital signs were good and she went home in good physical condition.

Two months later, she came for follow up. The tinnitus and the cervical pain had resolved majorly on the left side, and also her hearing was improved in her left ear. We performed the therapy of the vagal nerve in the same way at the right side. After two months, also the tinnitus and cervical pain on the right side was considerably improved. The intensity of the tinnitus was reduced to $5 \%$ of the original intensity at the left side and to $20 \%$ at the right side. Her hearing test are shown in Figure 1. An improved hearing of 20 to $30 \mathrm{~dB}$ from $250 \mathrm{~Hz}$ to 8 $\mathrm{kHz}$ in the left ear and from $250 \mathrm{~Hz}$ to $2 \mathrm{kHz}$ in the right ear was objectivated in the audiogram. Her life changed dramatically.

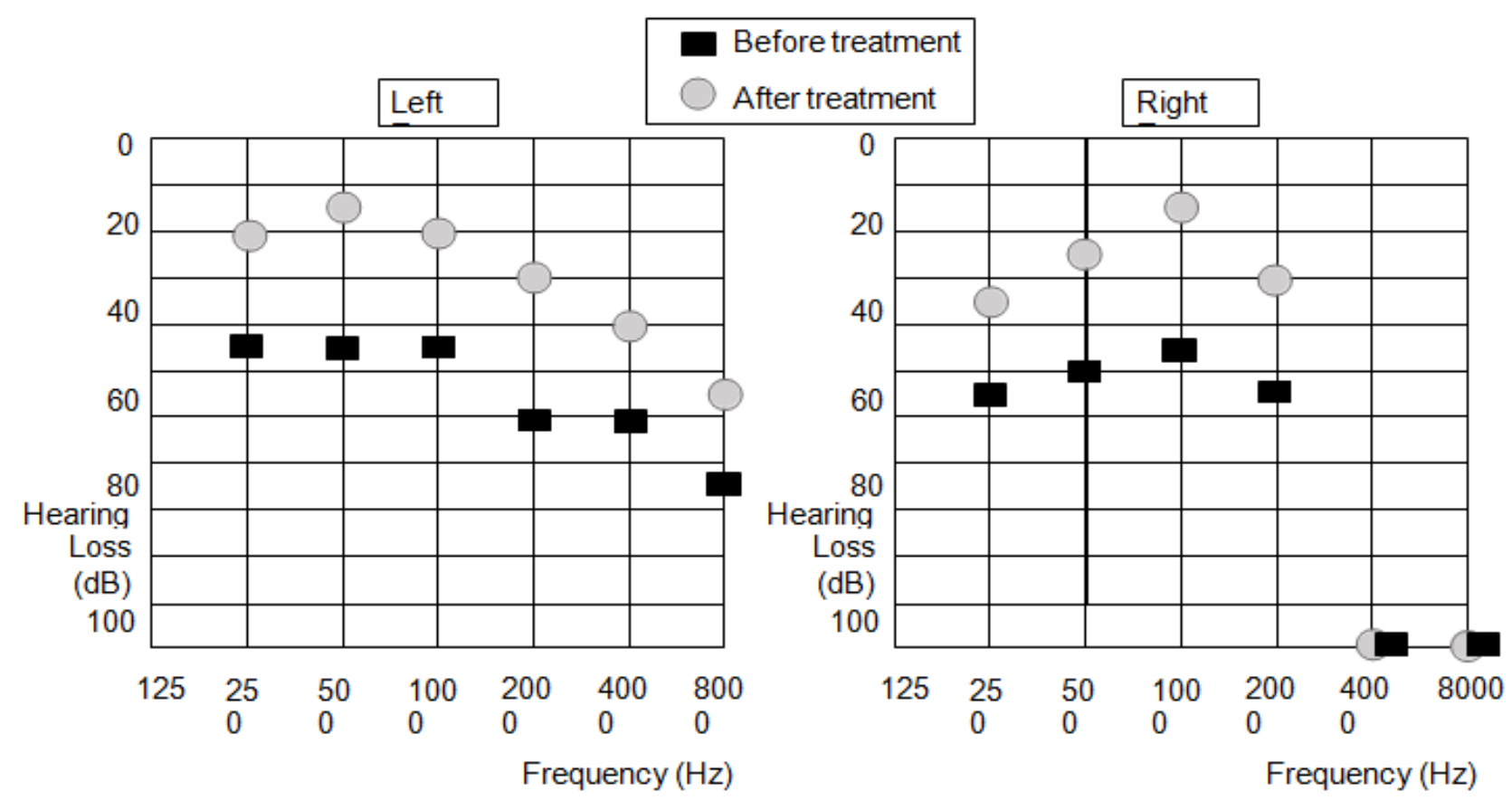

Figure 1: Hearing intensity before and after treatment. 


\section{DISCUSSION}

This case-study is one of the first to document that PRF of the vagal nerve can reduce tinnitus and improve hearing in a patient with severe tinnitus and hearing loss. The intensity of the tinnitus in both ears decreased considerably. An improved hearing of 20 to $30 \mathrm{~dB}$ from $250 \mathrm{~Hz}$ to $8 \mathrm{kHz}$ in the left ear and from $250 \mathrm{~Hz}$ to $2 \mathrm{kHz}$ in the right ear was objectivated in the audiogram.

Tinnitus may be the sequel of maladaptive interferences of neurotransmission in the central auditory pathway? Neuromodulation of brain structures related to tinnitus appeared to be a potential therapy of tinnitus patients ${ }^{8}$. The aim of VNS is to turnaround of pathological neuroplastic alterations of the auditory cortex toward normal neural activity and synchronicity ${ }^{9}$. This shift in neural performance should adjust the tinnitus percept and making it less annoying ${ }^{10}$. Stimulation of the ABVN is a good alternative for direct VNS. It has similar central auditory effect as implanted VNS, but it minimizes side effects and high costs.

Halting tinnitus by electrical impulse administration to the ear canal is a well-known therapy. The efficacy of this therapy is reported by some early studies and varies from 36 to $45 \%$ of patients noticing decreased intensity of their tinnitus ${ }^{11}$. Also, self-reported and audiometric hearing changed for the better in both groups, in $34 \%$ and $26 \%$ of ears, respectively. The positive outcome stayed after 30 and 90 days. The therapy did not induce hearing loss.

VNS may work by stimulating the nucleus of the solitary tract, which consequently activate the locus coeruleus and nucleus basalis, and they set free neuromodulators with notable effects in neurons in the cortex, hippocampus, and amygdala ${ }^{2}$. Especially, the muscarinic neuromodulation of the auditory cortex by the activation of nucleus basalis Meynerti is considered to be an important part of the VNS mechanism of action in chronic tinnitus patients ${ }^{9}$. The cholinergic neuromodulators may be involved in patients where hearing loss occurs with tinnitus or cognitive impairment and the cholinergic synapses between the medial olivocochlear efferent system and the outer hair cells are held responsible for it ${ }^{12}$. Therefore, the cholinergic effect of VNS may explain not only the beneficial effect on the intensity of tinnitus but also on the hearing capacity in the patient described in the case study.

PRF deliver brief pulses of radiofrequency to neural structures, and is a neuro-modulatory technique in the therapy of neuropathic pain. The intention of the deliverance of a high-frequency current in a pulse is to dissipate the heat generated in each active cycle during the long resting phase ${ }^{6}$. The temperature will not exceed $42^{\circ} \mathrm{C}$. This is differently from radiofrequency thermocoagulation, which harm pain fibres via a continuous temperature over $65^{\circ} \mathrm{C}$. PRF could be applied frequently without destruction of neuronal tissue. The stimulation of $A B V N$ is an easy and non-invasive method to obtain the beneficial effects of vagal system activation.

There are several limitations in our study. First, a backward-looking case report unavoidably has a selection bias. Thus, research with large patient groups is wanted to sanction our conclusions. Secondly, the longterm follow-up of PRF of the vagal nerve for tinnitus has not been assessed and also need further study.

\section{CONCLUSION}

This case-study document that PRF of the vagal nerve can reduce tinnitus and improve hearing in a patient with severe tinnitus and hearing loss. Neuromodulation of the auditory pathway appeared to be a potential therapy of tinnitus patients. The stimulation of the ABVN is an easy and non-invasive method to obtain the beneficial effects of vagal system activation. PRF is a minimally invasive neuromodulator technique that could be applied frequently without destruction of neuronal tissue. However, further research of PRF of the vagal nerve in tinnitus patients is needed.

\section{CONFLICT OF INTEREST}

The authors declares no conflict of interest

\section{REFERENCES}

1. Tyler R, Cacace A, Stocking C, Tarver B, Engineer N, Martin $J$, et al. Vagus nerve stimulation paired with tones for the treatment of tinnitus: a prospective randomized doubleblind controlled pilot study in humans. Scienti Reports. 2017;7(1):1-1.

2. Li TT, Wang ZJ, Yang SB, Zhu JH, Zhang SZ, Cai SJ, et al. Transcutaneous electrical stimulation at auricular acupoints innervated by auricular branch of vagus nerve pairing tone for tinnitus: study protocol for a randomized controlled clinical trial. Trials. 2015;16(1):1-9.

3. Yakunina N, Nam EC. Direct and Transcutaneous Vagus Nerve Stimulation for Treatment of Tinnitus: A Scoping Review. Front Neurosci. 2021;15:680590.

4. Ylikoski J, Markkanen M, Pirvola U, Lehtimäki JA, Ylikoski M, Jing Z, et al. Stress and Tinnitus; Transcutaneous Auricular Vagal Nerve Stimulation Attenuates Tinnitus-Triggered Stress Reaction. Frontiers in Psychology. 2020;11:2442.

5. Facchini G, Spinnato P, Guglielmi G, Albisinni U, Bazzocchi A. A comprehensive review of pulsed radiofrequency in the treatment of pain associated with different spinal conditions. The British J Radiol. 2017;90(1073):20150406.

6. Jia Y, Shrestha N, Wang X, Wang T, Luo F. The LongTerm Outcome of CT-Guided Pulsed Radiofrequency in the Treatment of Idiopathic Glossopharyngeal Neuralgia: A Retrospective Multi-Center Case Series. J Pain Res. 2020;13:2093.

7. Shore SE, Roberts LE, Langguth B. Maladaptive plasticity in tinnitus-triggers, mechanisms and treatment. Nature Rev Neurol. 2016;12(3):150-60. 
8. D'Arcy S, Hamilton C, Hughes S, Hall DA, Vanneste S, Langguth $\mathrm{B}$, et al. Bi-modal stimulation in the treatment of tinnitus: a study protocol for an exploratory trial to optimise stimulation parameters and patient subtyping. BMJ Open. 2017;7(10):e018465.

9. Bojić T, Perović VR, Senćanski M, Glišić S. Identification of candidate allosteric modulators of the $\mathrm{m} 1$ muscarinic acetylcholine receptor which may improve vagus nerve stimulation in chronic tinnitus. Frontiers in Neurosci. 2017; $11: 636$.
10. Hays SA, Rennaker RL, Kilgard MP. Targeting plasticity with vagus nerve stimulation to treat neurological disease. Progress in Brain Res. 2013;207:275-299.

11. Hoare DJ, Adjamian P, Sereda M. Electrical stimulation of the ear, head, cranial nerve, or cortex for the treatment of tinnitus: a scoping review. Neural plasticity. 2016.

12. Ruan Q, Yu Z, Zhang W, Ruan J, Liu C, Zhang R. Cholinergic hypofunction in presbycusis-related tinnitus with cognitive function impairment: emerging hypotheses. Frontiers in Aging Neurosci. 2018;10:98. 